\title{
A Game-Theoretic Analysis of Bandwidth Allocation under a User-Grouping Constraint
}

\author{
Shun-Pin Hsu, ${ }^{1}$ Shun-Liang Hsu, ${ }^{2}$ and Alan Shenghan Tsai ${ }^{1}$ \\ ${ }^{1}$ Department of Electrical Engineering, National Chung Hsing University, 250 Kuo-Kuang Road, Taichung 402, Taiwan \\ ${ }^{2}$ Department of Law and Graduate Institute of Technology Management, National Chung Hsing University, 250 Kuo-Kuang Road, \\ Taichung 402, Taiwan
}

Correspondence should be addressed to Shun-Pin Hsu; shsu@nchu.edu.tw

Received 2 October 2012; Revised 22 December 2012; Accepted 26 December 2012

Academic Editor: Xiaoning Zhang

Copyright (C) 2013 Shun-Pin Hsu et al. This is an open access article distributed under the Creative Commons Attribution License, which permits unrestricted use, distribution, and reproduction in any medium, provided the original work is properly cited.

\begin{abstract}
A new bandwidth allocation model is studied in this paper. In this model, a system, such as a communication network, is composed of a finite number of users, and they compete for limited bandwidth resources. Each user adopts the decision that maximizes his or her own benefit characterized by the utility function. The decision space of each user is subject to constraints. In addition, some users form a group, and their joint decision space is also subject to constraints. Under the assumption that each user's utility function satisfies some continuity and concavity conditions, the existence, uniqueness, and fairness, in some appropriate sense, of the Nash equilibrium point in the allocation game are proved. An algorithm yielding a sequence converging to the equilibrium point is proposed. Finally, a numerical example with detailed analysis is provided to illustrate the effectiveness of our work.
\end{abstract}

\section{Introduction}

With the widespread use of internet and the increasing popularity of mobile devices, more and more people can get online at almost anytime and anywhere. An immediate challenge facing the significant increment of online users is the support of quality of service (QoS). Over the past decade considerable efforts have been made to ensure the smooth operation of the networking systems. For example, the load balancing problems were considered by Anselmi et al. [1] and Ayesta et al. [2]. The routing problems were studied by La and Anantharam [3], Richman and Shimkin [4], Boulogne et al. [5], and Korilis et al. [6-8]. Niyato and Hossain [9] studied the practical issue such as the admission control for the wireless broadband standard. Ganesh et al. [10] and Yaïche et al. [11] considered the pricing issues. In modeling the networking problems, quite often the bandwidth availability is the main concern and has its role in the associated performance measure. The network system quantifies the results caused by different operation scenarios and seeks the approach leading to the greatest benefits in some sense. Since the benefit of any network user inevitably involves that of other users, its evaluation is mostly carried out in the context of game theory. In the survey paper Altman et al. collected a long list of networking models based on game theoretic formulation. Interested readers are referred to [12] and the rich reference therein.

As mentioned above, the bandwidth availability is the major concern in many networking problems. The bandwidth allocation is thus the core issue as far as the quality of service is concerned. While the bandwidth allocation problem was considered by many authors in different contexts of networking protocols or communication standards (see e.g., $[9,11,13$, $14]$ ), at a high level of abstraction the problem can be regarded as the classical resources distribution problem studied in many professional fields such as economics, management science, and operations research. Given a finite number of units competing for the limited resources, how does each unit decide its share based on its own utility function? Lazar et al. [15] formulated this problem for the network composed of noncooperative users. Under certain monotonicity, differentiability, and convexity assumptions on the cost function the unique existence and certain fairness property of the Nash equilibrium point (NEP) were proved. An algorithm based on 
Gauss-Seidel and Jacobi schemes was proposed and proved to yield a sequence converging to the NEP. However, the framework in [15] assumes only the natural constraint for the bandwidth allocation. That is, the feasible bandwidth of any user falls within the interval lower bounded by zero and upper bounded by the bandwidth available for that user. In practice some techniques such as the bandwidth throttling and bandwidth/traffic shaping [16] are available to provide more adaptive bandwidth control. To address this issue, Rhee and Konstantopoulos [17, 18] relaxed the assumption and allowed some prespecified numbers for the upper and lower bounds of the bandwidth. This relaxation increases the flexibility of flow control and helps the QoS satisfaction by the networking system. On the other hand, in modern broadband communication systems some users might form a group and expect the group-wise QoS, in addition to the user-wise one. For example, the customers of an internet service provider (ISP) might include the individuals and a company with many employees. To maintain the QoS, parameters would be assigned to bound the bandwidth of each individual and each employee. Furthermore the ISP and the company would set the constraint for the employee-averaged, or equivalently, employee-totaled bandwidth, as shown in Figure 1. A similar concept of group constraint can be seen in the cost-effective broadband access network such as the Ethernet-based passive optical network (EPON) $[19,20]$. This system is composed of an optical line terminal (OLT) and many optical network units (ONUs). The OLT is situated in the central office and ONUs are distributed over the remote areas for multimedia communication with the subscribers. In the upload process each ONU adopts the time division multiplexing access (TDMA) protocol to transmit data frames to the OLT, in the sense that each ONU only transmits the data during the time slots specifically scheduled for it [21]. The protocol avoids the frame collisions between different ONUs at the cost of imposing the upper bound for the time-average flow of each ONU and the upper bound for the total flow of all ONUs.

In light of the bandwidth sharing mechanism in EPON and other similar systems, we extend the existing results to include the user-grouping constraint for better model fitting. Suppose each user has his or her own utility function that describes the relation between the allocated bandwidth and the resultant benefit to that user. Following the standard assumptions, (1) the function depends on the bandwidth of the user, and on the bandwidth of other users only through their total bandwidth, and (2) the function satisfies certain continuity and concavity properties; we show the unique existence and the fairness with some appropriate sense, of the NEP in the allocation game. The contributions of our work are twofold. First, a novel concept called usergrouping NEP is proposed. This concept is corresponding to the new introduction of the group constraint, under which the uniqueness of NEP proved in $[15,17]$ no longer holds. Based on this concept we give a new definition for the equilibrium point and prove its uniqueness under our assumptions. The fairness of the allocation based on the user-grouping NEP is also proved. Second, we show that the Gauss-Seidel type algorithm in $[15,18]$ can be modified to yield a sequence converging to the user-grouping NEP. Since

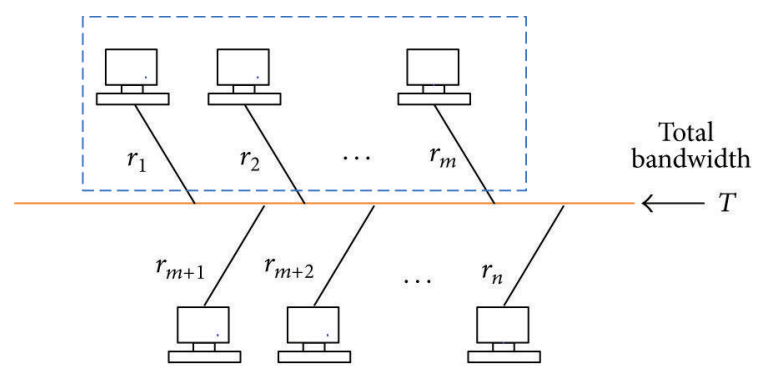

FIgURE 1: The system bandwidth allocation $\mathbf{r}=\left(r_{1}, r_{2}, \ldots, r_{n}\right)$ subject to user-wise constraints: $\underline{r}_{i} \leq r_{i} \leq \bar{r}^{i}$ for $i \in\{1,2, \ldots, n\}$, and a user-grouping constraint $\underline{R}_{g} \leq \sum_{i=1}^{m} r_{i} \leq \bar{R}_{g}$. The total allocation $R=\sum_{i=1}^{n} r_{i}$ is less than $T$, the total bandwidth.

the bandwidth allocation is of central concern in networking systems, our results might result in the reinvestigation and reformulation of other networking issues such that more practical approaches can be developed.

\section{Preliminaries}

Suppose a networking system has $n$ users and they compete for the system bandwidth. Each user is assigned with the bandwidth subject to predecided upper and lower bounds. In addition, $m$ of the $n$ users form a group and the total bandwidth of the $m$ users is also subject to a predecided constraint. We would like to design the system bandwidth allocation policy that optimizes the performance index of each user in the game-theoretical sense. For convenience, we use the list of nomenclature shown at the end of the paper.

Assume the utility function for each user depends on the bandwidth of that user and the total bandwidth of other users. That is, the utility function for user $i$ in $\mathcal{N}$ can be written as $U_{i}\left(r_{i}, R\right)$. Also, assume the utility function satisfies the following continuity and concavity properties [18].

Assumption 1. For each utility function $U_{i}\left(r_{i}, R\right)$

(a) $U_{i}\left(r_{i}, R\right)$ is continuously differentiable with respect to $r_{i}$

(b) $\left(\partial / \partial r_{i}\right) U_{i}\left(r_{i}, R\right)$ is strictly decreasing with respect to $r_{i}$ and nonincreasing with respect to $R$.

Now we define the allocation function. For user $i$ in $\mathscr{N} \backslash \mathscr{M}$ with the available bandwidth $T_{i}$, the allocation function $A_{i}$ is defined as

$$
A_{i}\left(T_{i}\right)=\arg \max _{\underline{r}_{i} \leq r \leq \bar{r}_{i}} U_{i}\left(r, r+T-T_{i}\right) .
$$

For user $i$ in $\mathscr{M}$ with the available bandwidth $T_{i}$ and insidegroup information $T_{i}^{g}$, the allocation function $\mathscr{A}_{i}$ is defined as

$$
\mathscr{A}_{i}\left(T_{i}, T_{i}^{g}\right)=\arg \max _{\underline{r}_{i} \leq r \leq \bar{r}_{i}, \underline{R}_{g} \leq T_{i}^{g}+r \leq \bar{R}_{g}} U_{i}\left(r, r+T-T_{i}\right) .
$$


Assumption 2. The bandwidth allocation with the constraint parameters has the following properties:

(a) $T_{i}>\mathscr{A}_{i}\left(T_{i}, T_{i}^{g}\right)$ for each feasible $T_{i}$ and $T_{i}^{g}$ where $i \in \mathscr{M}$, and $T_{i}>A_{i}\left(T_{i}\right)$ for each feasible $T_{i}$ where $i \in \mathcal{N} \backslash \mathscr{M}$;

(b) $\sum_{i=1}^{m} \bar{r}_{i}>\bar{R}_{g}>\underline{R}_{g}>\sum_{i=1}^{m} \underline{r}_{i}$.

In our framework, the classical Nash equilibrium point (NEP) in the allocation game is defined as

$$
\mathbf{r}^{*}:=\left(r_{1}^{*}, r_{2}^{*}, \ldots, r_{n}^{*}\right)
$$

where

$$
r_{i}^{*} \in \arg \max _{r \in C_{i}} U_{i}\left(r, r+\sum_{j \neq i} r_{j}^{*}\right) .
$$

Note that $C_{i}$ is $\left\{r \mid \underline{r}_{i} \leq r \leq \bar{r}_{i}, \underline{R}_{g} \leq r+\sum_{j \in \mathscr{M} \backslash\{i\}} r_{j}^{*} \leq \bar{R}_{g}\right\}$ if $i \in \mathscr{M}$ and is $\left\{r \mid \underline{r}_{i} \leq r \leq \bar{r}_{i}\right\}$ otherwise. The user-grouping NEP is defined as

$$
\mathbf{r}_{g}^{*}:=\left(\frac{R_{g}^{*}}{m}, r_{m+1}^{*}, r_{m+2}^{*}, \ldots, r_{n}^{*}\right),
$$

where

$$
R_{g}^{*}:=\sum_{i=1}^{m} r_{i}^{*}
$$

Remark 3. The definitions (3)-(4) reflect the central concept of the well-studied constrained NEP. That is, given the constrained strategy space $C_{i}$ for each user $i \in \mathcal{N}, r_{i}^{*}$ is defined as the maximizer of the utility function of user $i$ provided that the strategy $r_{j}^{*}$ is adopted by user $j$ for each $j \in \mathcal{N} \backslash\{i\}$. Note that the bandwidth of users in the group $\mathscr{M}$ should satisfy the extra group constraint and thus $r_{i}^{*}$ might lose its uniqueness in $C_{i}$ as the group constraint is active. The novel concept of user-grouping NEP in (5)-(6) is thus proposed to compensate the property of the equilibrium point. We will show in Section 3.1 that our setting ensures the uniqueness of the user-grouping NEP.

Remark 4. Suppose $T_{i}^{*}:=T-\sum_{j \neq i}^{n} r_{j}^{*}$. Also, let $T_{i}^{g *}:=\bar{R}_{g}-$ $\sum_{j \neq i}^{m} r_{j}^{*}$, then

$$
r_{i}^{*}:= \begin{cases}\mathscr{A}_{i}\left(T_{i}^{*}, T_{i}^{g *}\right) & \text { if } i \in \mathscr{M} \\ A_{i}\left(T_{i}^{*}\right) & \text { if } i \in \mathscr{N} \backslash \mathscr{M} .\end{cases}
$$

By part (a) in Assumption 2 we have

$$
r_{i}^{*}<T_{i}^{*}=T-\sum_{j \neq i}^{n} r_{j}^{*} .
$$

Consequently,

$$
R^{*}:=\sum_{i=1}^{n} r_{i}^{*}<T
$$

This means that the NEP, or $\mathbf{r}^{*}$, satisfies the natural constraint $R^{*} \leq T$ and the constraint is always inactive. Part (b) in Assumption 2 is a natural condition such that the constraint $\underline{R}_{g} \leq \sum_{i=1}^{m} r_{i} \leq \bar{R}_{g}$ makes sense.

The existence of the NEP in our setting is guaranteed by Rosen's result in the following.

Theorem 5 (see [22, Theorem 1]). An equilibrium point exists for every concave n-person game.

Theorem 5 can be obtained using the classical Kakutani fixed point theorem and in some sense generalizes Nash's setting on the strategy space of the users [23, 24]. In the next section we delve into other properties and propose an algorithm to locate the NEP.

\section{Main Results}

3.1. Uniqueness. Our first result is concerned with the uniqueness of the user-grouping NEP. This property as shown in [18, page 13] is not implied by the uniqueness theorem in [22]. For the NEP $\mathbf{r}^{*}=\left(r_{1}^{*}, r_{2}^{*}, \ldots, r_{n}^{*}\right)$ defined in (3)-(4), the Karash-Kuhn-Tucker (KKT) conditions must be satisfied. That is, for each $i \in \mathscr{N} \backslash \mathscr{M}$ there exist KKT multipliers $\bar{\lambda}_{i}^{*}$ and $\underline{\lambda}_{i}^{*}$ (see e.g., [25, page 458]) such that

$$
\begin{gathered}
-\frac{\partial}{\partial r_{i}} U_{i}\left(r_{i}^{*}, R^{*}\right)+\bar{\lambda}_{i}^{*}-\underline{\lambda}_{i}^{*}=0, \\
\bar{\lambda}_{i}^{*}\left(r_{i}^{*}-\bar{r}_{i}\right)=0, \\
\underline{\lambda}_{i}^{*}\left(\underline{r}_{i}-r_{i}^{*}\right)=0, \\
\underline{r}_{i} \leq r_{i}^{*} \leq \bar{r}_{i}, \\
\underline{\lambda}_{i}^{*} \geq 0, \quad \bar{\lambda}_{i}^{*} \geq 0 .
\end{gathered}
$$

In addition, for each $i \in \mathscr{M}$ there exist KKT multipliers $\bar{\lambda}_{i}^{*}$ and $\underline{\lambda}_{i}^{*}$ satisfying (11)-(14), and $\bar{\gamma}_{i}^{*}$ and $\underline{\gamma}_{i}^{*}$ satisfying

$$
\begin{gathered}
-\frac{\partial}{\partial r_{i}} U_{i}\left(r_{i}^{*}, R^{*}\right)+\bar{\lambda}_{i}^{*}-\underline{\lambda}_{i}^{*}+\bar{\gamma}_{i}^{*}-\underline{\gamma}_{i}^{*}=0, \\
\bar{\gamma}_{i}^{*}\left(R_{g}^{*}-\bar{R}_{g}\right)=0, \\
\underline{\gamma}_{i}^{*}\left(\underline{R}_{g}-R_{g}^{*}\right)=0, \\
\underline{R}_{g} \leq R_{g}^{*} \leq \bar{R}_{g}, \\
\underline{\gamma}_{i}^{*} \geq 0, \quad \bar{\gamma}_{i}^{*} \geq 0
\end{gathered}
$$

where $R_{g}^{*}$ is defined in (6).

Lemma 6. For each $i \in \mathscr{N} \backslash \mathscr{M}$, let $r_{i}^{(1)}:=A_{i}\left(T_{i}^{(1)}\right)$ and $r_{i}^{(2)}:=$ $A_{i}\left(T_{i}^{(2)}\right)$ for some feasible $T_{i}^{(1)}$ and $T_{i}^{(2)}$, then

$$
r_{i}^{(1)}>r_{i}^{(2)} \Longrightarrow \bar{\lambda}_{i}^{(1)}-\underline{\lambda}_{i}^{(1)} \geq \bar{\lambda}_{i}^{(2)}-\underline{\lambda}_{i}^{(2)},
$$


where the nonnegative KKT multipliers $\bar{\lambda}_{i}^{(1)}, \underline{\lambda}_{i}^{(1)}, \bar{\lambda}_{i}^{(2)}$ and $\underline{\lambda}_{i}^{(2)}$ satisfy

$$
\begin{array}{ll}
\bar{\lambda}_{i}^{(1)}\left(r_{i}^{(1)}-\bar{r}_{i}\right)=0, & \underline{\lambda}_{i}^{(1)}\left(\underline{r}_{i}-r_{i}^{(1)}\right)=0, \\
\bar{\lambda}_{i}^{(2)}\left(r_{i}^{(2)}-\bar{r}_{i}\right)=0, & \underline{\lambda}_{i}^{(2)}\left(\underline{r}_{i}-r_{i}^{(2)}\right)=0 .
\end{array}
$$

Proof. Since $r_{i}^{(1)}$ and $r_{i}^{(2)}$ are both feasible, $r_{i}^{(1)}>r_{i}^{(2)}$ implies $\underline{\lambda}_{i}^{(1)}=0$ and $\bar{\lambda}_{i}^{(2)}=0$ by (21) and (22), respectively. Consequently, the result follows since $\bar{\lambda}_{i}^{(1)} \geq 0$ and $\underline{\lambda}_{i}^{(2)} \geq 0$.

Theorem 7. The user-grouping NEP defined in (5) is unique.

Proof. Suppose $\mathbf{r}^{(1)}=\left(r_{1}^{(1)}, r_{2}^{(1)}, \ldots, r_{n}^{(1)}\right)$ and $\mathbf{r}^{(2)}=\left(r_{1}^{(2)}, r_{2}^{(2)}\right.$, $\left.\ldots, r_{n}^{(2)}\right)$ are both the equilibrium points. Let $R^{(1)}=\sum_{i=1}^{n} r_{i}^{(1)}$ and $R^{(2)}=\sum_{i=1}^{n} r_{i}^{(2)}$. We can thus write for $i \in \mathscr{N} \backslash \mathscr{M}$ that

$$
\begin{aligned}
& -\frac{\partial}{\partial r_{i}} U_{i}\left(r_{i}^{(1)}, R^{(1)}\right)+\bar{\lambda}_{i}^{(1)}-\underline{\lambda}_{i}^{(1)}=0, \\
& -\frac{\partial}{\partial r_{i}} U_{i}\left(r_{i}^{(2)}, R^{(2)}\right)+\bar{\lambda}_{i}^{(2)}-\underline{\lambda}_{i}^{(2)}=0,
\end{aligned}
$$

where $\bar{\lambda}_{i}^{(1)}, \underline{\lambda}_{i}^{(1)}, \bar{\lambda}_{i}^{(2)}, \underline{\lambda}_{i}^{(2)}$ are the associated KKT multipliers. Assume that $R^{(1)}>R^{(2)}$. If there exists $i \in \mathscr{N} \backslash \mathscr{M}$ such that $r_{i}^{(1)}>r_{i}^{(2)}$, by Lemma 6 and Assumption 1 we have

$$
\begin{aligned}
-\frac{\partial}{\partial r_{i}} U_{i}\left(r_{i}^{(1)}, R^{(1)}\right)+\bar{\lambda}_{i}^{(1)}-\underline{\lambda}_{i}^{(1)} & \\
& >-\frac{\partial}{\partial r_{i}} U_{i}\left(r_{i}^{(2)}, R^{(2)}\right)+\bar{\lambda}_{i}^{(2)}-\underline{\lambda}_{i}^{(2)},
\end{aligned}
$$

which is a contradiction. We thus have $r_{i}^{(1)} \leq r_{i}^{(2)}$ for $i$ in $\mathscr{N} \backslash \mathscr{M}$. This implies $R_{g}^{(1)}:=\sum_{i=1}^{m} r_{i}^{(1)}>\sum_{i=2}^{m} r_{i}^{(2)}=R_{g}^{(2)}$. Note that, for $i \in \mathscr{M}$,

$$
\begin{aligned}
& -\frac{\partial}{\partial r_{i}} U_{i}\left(r_{i}^{(1)}, R^{(1)}\right)+\bar{\lambda}_{i}^{(1)}-\underline{\lambda}_{i}^{(1)}+\bar{\gamma}_{i}^{(1)}-\underline{\gamma}_{i}^{(1)}=0, \\
& -\frac{\partial}{\partial r_{i}} U_{i}\left(r_{i}^{(2)}, R^{(2)}\right)+\bar{\lambda}_{i}^{(2)}-\underline{\lambda}_{i}^{(2)}+\bar{\gamma}_{i}^{(2)}-\underline{\gamma}_{i}^{(2)}=0 .
\end{aligned}
$$

With similar arguments in proving Lemma 6 we can show that

$$
R_{g}^{(1)}>R_{g}^{(2)} \Longrightarrow \bar{\gamma}_{i}^{(1)}-\underline{\gamma}_{i}^{(1)} \geq \bar{\gamma}_{i}^{(2)}-\underline{\gamma}_{i}^{(2)} .
$$

If $r_{i}^{(1)}>r_{i}^{(2)}$ for some $i \in \mathscr{M}$, we have

$$
\begin{aligned}
-\frac{\partial}{\partial r_{i}} U_{i}\left(r_{i}^{(1)}, R^{(1)}\right)+\bar{\lambda}_{i}^{(1)}-\underline{\lambda}_{i}^{(1)}+\bar{\gamma}_{i}^{(1)}-\underline{\gamma}_{i}^{(1)} \\
\quad>-\frac{\partial}{\partial r_{i}} U_{i}\left(r_{i}^{(2)}, R^{(2)}\right)+\bar{\lambda}_{i}^{(2)}-\underline{\lambda}_{i}^{(2)}+\bar{\gamma}_{i}^{(2)}-\underline{\gamma}_{i}^{(2)},
\end{aligned}
$$

which is a contradiction. We thus have $r_{i}^{(i)} \leq r_{i}^{(2)}$ for each $i \in \mathscr{M}$ and therefore $R_{g}^{(1)} \leq R_{g}^{(2)}$, also a contradiction. With analogous arguments we can show that assuming $R^{(1)}<R^{(2)}$ also leads to a contradiction. Therefore $R^{(1)}=R^{(2)}$ and by Lemma $6 r_{i}^{(1)}=r_{i}^{(2)}$ for $i \in \mathcal{N} \backslash \mathscr{M}$. This implies $R_{g}^{(1)}=R_{g}^{(2)}$; therefore $\mathbf{r}_{g}^{*}$ is unique.

3.2. Fairness. A bandwidth allocation $\mathbf{r}=\left(r_{1}, r_{2}, \ldots, r_{n}\right)$ is said to be fair if for any feasible $t_{1}$ and $t_{2}$

$$
\mathscr{A}_{i}\left(t_{1}, t_{2}\right) \geq \mathscr{A}_{j}\left(t_{1}, t_{2}\right) \Longrightarrow r_{i} \geq r_{j},
$$

where $i, j \in \mathscr{M}$, and for any feasible $t$

$$
A_{i}(t) \geq A_{j}(t) \Longrightarrow r_{i} \geq r_{j},
$$

where $i, j \in \mathcal{N} \backslash \mathscr{M}$. This definition suggests that a fair allocation guarantees the user in greater need of bandwidth actually obtains more bandwidth.

Theorem 8. The bandwidth allocation based on the NEP defined in (3)-(4) is fair.

Proof. Suppose $i \in \mathscr{M}$. Let $r_{i}^{(1)}=\mathscr{A}_{i}\left(T_{i}^{(1)}, t\right)$ and $r_{i}^{(2)}=$ $\mathscr{A}_{i}\left(T_{i}^{(1)}+\Delta T, t+\Delta T\right)$. We thus have

$$
\begin{aligned}
& -\frac{\partial}{\partial r_{i}} U_{i}\left(r_{i}^{(1)}, R^{(1)}\right)+\bar{\lambda}_{i}^{(1)}-\underline{\lambda}_{i}^{(1)}+\bar{\gamma}_{i}^{(1)}-\underline{\gamma}_{i}^{(1)}=0, \\
& -\frac{\partial}{\partial r_{i}} U_{i}\left(r_{i}^{(2)}, R^{(2)}\right)+\bar{\lambda}_{i}^{(2)}-\underline{\lambda}_{i}^{(2)}+\bar{\gamma}_{i}^{(2)}-\underline{\gamma}_{i}^{(2)}=0 .
\end{aligned}
$$

Assume $r_{i}^{(2)}-\Delta T>r_{i}^{(1)}$, which implies $r_{i}^{(2)}>r_{i}^{(1)}$ and by Lemma $6 \bar{\lambda}_{i}^{(2)}-\underline{\lambda}_{i}^{(2)} \geq \bar{\lambda}_{i}^{(1)}-\underline{\lambda}_{i}^{(1)}$. Also,

$$
\begin{aligned}
R^{(2)} & :=r_{i}^{(2)}+R_{-i}^{(2)}>r_{i}^{(1)}+\Delta T+R_{-i}^{(2)} \\
& =r_{i}^{(1)}+R_{-i}^{(1)}:=R^{(1)} .
\end{aligned}
$$

Similarly,

$$
\begin{aligned}
R_{g}^{(2)} & :=r_{i}^{(2)}+\sum_{j \neq i}^{m} r_{j}^{(2)}>r_{i}^{(1)}+\Delta T+\sum_{j \neq i}^{m} r_{j}^{(2)} \\
& =r_{i}^{(1)}+\sum_{j \neq i}^{m} r_{j}^{(1)}:=R_{g}^{(1)} .
\end{aligned}
$$

Note that (32) implies $\bar{\gamma}_{i}^{(2)}-\underline{\gamma}_{i}^{(2)} \geq \bar{\gamma}_{i}^{(1)}-\underline{\gamma}_{i}^{(1)}$. We then have

$$
\begin{aligned}
-\frac{\partial}{\partial r_{i}} U_{i}\left(r_{i}^{(2)}, r_{i}^{(2)}+R_{-i}^{(2)}\right)+\bar{\lambda}_{i}^{(2)}-\underline{\lambda}_{i}^{(2)}+\bar{\gamma}_{i}^{(2)}-\underline{\gamma}_{i}^{(2)} \\
>-\frac{\partial}{\partial r_{i}} U_{i}\left(r_{i}^{(1)}, r_{i}^{(1)}+R_{-i}^{(1)}\right)+\bar{\lambda}_{i}^{(1)}-\underline{\lambda}_{i}^{(1)}+\bar{\gamma}_{i}^{(1)}-\underline{\gamma}_{i}^{(1)},
\end{aligned}
$$

which is a contradiction. Therefore, $r_{i}^{(2)}-\Delta T \leq r_{i}^{(1)}$, which implies

$$
T_{i}^{(1)}+\Delta T-r_{i}^{(2)} \geq T_{i}^{(1)}-r_{i}^{(1)},
$$


namely,

$$
T_{i}^{(1)}+\Delta T-\mathscr{A}_{i}\left(T_{i}^{(1)}+\Delta T, t+\Delta T\right) \geq T_{i}^{(1)}-\mathscr{A}_{i}\left(T_{i}^{(1)}, t\right) .
$$

To show the allocation based on $\mathbf{r}^{*}$ is fair, consider first the case that $i, j \in \mathscr{M}$. By definition $r_{i}^{*}=\mathscr{A}_{i}\left(T_{i}^{*}, T_{i}^{g^{*}}\right), r_{j}^{*}:=$ $\mathscr{A}_{j}\left(T_{j}^{*}, T_{j}^{g *}\right)$ where $T_{i}^{*}, T_{i}^{g *}, T_{j}^{*}$ and $T_{j}^{g *}$ are all feasible. With $R^{*}$ defined in (9) we have

$$
T-R^{*}=T_{i}^{*}-\mathscr{A}_{i}\left(T_{i}^{*}, T_{i}^{g *}\right)=T_{j}^{*}-\mathscr{A}_{j}\left(T_{j}^{*}, T_{i}^{g *}\right),
$$

which equals the remaining bandwidth of the system after allocation. Given $\mathscr{A}_{i}\left(T_{i}^{*}, T_{i}^{g^{*}}\right)>\mathscr{A}_{j}\left(T_{i}^{*}, T_{i}^{g^{*}}\right)$, we have

$$
\begin{aligned}
T_{i}^{*}-\mathscr{A}_{j}\left(T_{i}^{*}, T_{i}^{g *}\right) & >T_{i}^{*}-\mathscr{A}_{i}\left(T_{i}^{*}, T_{i}^{g *}\right) \\
& =T_{j}^{*}-\mathscr{A}_{j}\left(T_{j}^{*}, T_{j}^{g *}\right) .
\end{aligned}
$$

Note that

$$
T_{i}^{*}-T_{j}^{*}=T_{i}^{g *}-T_{j}^{g *}=r_{i}^{*}-r_{j}^{*}:=\Delta T^{*} .
$$

Equations (35) and (37) imply $\Delta T^{*}>0$; hence $r_{i}^{*}>r_{j}^{*}$. The case for $i, j \in \mathscr{N} \backslash \mathscr{M}$ can be similarly proved and is thus ignored (see [18, Theorem 2.3] for details).

3.3. Algorithm. In this section we analyze the scheme to identify the user-grouping Nash equilibrium point. We say an individual update is implemented on user $i$ if the bandwidth of each user other than $i$ is fixed and the bandwidth of user $i$ is updated to maximize his or her utility function. In addition, we say a batch update occurs in the collection $\mathscr{K}$ of users if the bandwidth of each user not in $\mathscr{K}$ is fixed and the individual update is sequentially implemented on each user in $\mathscr{K}$ repeatedly till an equilibrium is reached. Here $\mathscr{K}$ is either $\mathscr{M}$ or $\mathscr{N} \backslash \mathscr{M}$. If $\mathscr{K}=\mathscr{M}$ the batch update is implemented assuming no group constraint, namely, $\bar{R}_{g}=\infty$ and $\underline{R}_{g}=0$. Note that the batch update is guaranteed to reach an equilibrium (see [15] and [18, Section 2.4]). As a result, suppose $\mathbf{r}^{k}=\left(r_{1}^{k}, r_{1}^{k}, \ldots, r_{n}^{k}\right)$ is the system allocation at step $k$. If an individual update occurs at user $i \in \mathscr{N} \backslash \mathscr{M}$ at step $k+1$, that means $r_{j}^{k+1}=r_{j}^{k}$ for $j \in \mathcal{N} \backslash\{i\}$, and

$$
-\frac{\partial}{\partial r_{i}} U_{i}\left(r_{i}^{k+1}, R^{k+1}\right)+\bar{\lambda}_{i}^{k+1}-\underline{\lambda}_{i}^{k+1}=0
$$

for some KKT multipliers $\bar{\lambda}_{i}^{k+1}$ and $\bar{\lambda}_{i}^{k+1}$ and $R^{k+1}=\sum_{i=1}^{n} r_{i}^{k+1}$. If a batch update occurs at the group of users at step $k+1$, that means $r_{j}^{k+1}=r_{j}^{k}$ for each $j \in \mathscr{N} \backslash \mathscr{M}$, and we can write for each $i \in \mathscr{M}$

$$
-\frac{\partial}{\partial r_{i}} U_{i}\left(r_{i}^{k+1}, R^{k+1}\right)+\bar{\lambda}_{i}^{k+1}-\underline{\lambda}_{i}^{k+1}+\bar{\gamma}_{i}^{k+1}-\underline{\gamma}_{i}^{k+1}=0,
$$

where $\bar{\lambda}_{i}^{k+1}, \bar{\lambda}_{i}^{k+1}, \bar{\gamma}_{i}^{k+1}$ and $\bar{\gamma}_{i}^{k+1}$ are the associated KKT multipliers. We now show that a repeated implementation of sequential batch updates on users in $\mathscr{M}$ and users in $\mathscr{N} \backslash \mathscr{M}$, as outline in Algorithm 1, yields a sequence converging to the user-grouping NEP $\mathbf{r}_{g}^{*}$ in (5). Define first the error measure $e\left(\mathbf{r}_{g}^{k}\right)$ between $\mathbf{r}_{g}^{k}$ and $\mathbf{r}_{g}^{*}$ as

$$
e\left(\mathbf{r}_{g}^{k}\right)=\sum_{i=m+1}^{n}\left|r_{i}^{k}-r_{i}^{*}\right|+\left|R_{g}^{k}-R_{g}^{*}\right|+\left|R^{k}-R^{*}\right|,
$$

where $R_{g}^{k}:=\sum_{i=1}^{m} r_{i}^{k} \cdot R_{g}^{*}$ and $R^{*}$ are defined in (6) and (9), respectively.

Lemma 9. The error measure $e\left(\mathbf{r}_{g}^{k}\right)$ defined in (41) is nonincreasing, namely, $e\left(\mathbf{r}_{g}^{k+1}\right) \leq e\left(\mathbf{r}_{g}^{k}\right)$ for any positive integer $k$.

Proof. If at step $k+1$ an individual update occurs at $i \epsilon$ $\mathscr{N} \backslash \mathscr{M},(39)$ is satisfied. Since (10) is also satisfied, we have by Lemma 6 and part (b) in Assumption 1 that

$$
\begin{aligned}
& R^{k+1} \geq R^{*} \Longrightarrow r_{i}^{k+1} \leq r_{i}^{*}, \\
& R^{k+1} \leq R^{*} \Longrightarrow r_{i}^{k+1} \geq r_{i}^{*} .
\end{aligned}
$$

Under the assumption

$$
\begin{aligned}
e\left(\mathbf{r}_{g}^{k+1}\right)-e\left(\mathbf{r}_{g}^{k}\right)= & \left|r_{i}^{k+1}-r^{*}\right|-\left|r_{i}^{k}-r^{*}\right| \\
& +\left|R^{k+1}-R^{*}\right|-\left|R^{k}-R^{*}\right| .
\end{aligned}
$$

Suppose $R^{k+1} \geq R^{*}$. If $R^{k} \geq R^{k+1}$, then

$$
\begin{aligned}
e\left(\mathbf{r}_{g}^{k+1}\right)-e\left(\mathbf{r}_{g}^{k}\right) & =-\left(r_{i}^{k+1}-r^{*}\right)-\left|r_{i}^{k}-r^{*}\right|+R^{k+1}-R^{k} \\
& \Longrightarrow \begin{cases}=0 & \text { if } r_{i}^{k}<r_{i}^{*} \\
<0 & \text { o.w. }\end{cases}
\end{aligned}
$$

Since $R^{k}<R^{k+1}$ implies $r_{i}^{k}<r_{i}^{k+1}$, we have

$$
\begin{aligned}
e\left(\mathbf{r}_{g}^{k+1}\right)-e\left(\mathbf{r}_{g}^{k}\right) & =-r_{i}^{k+1}+r_{i}^{k}+R^{k+1}-R^{*}-\left|R^{k}-R^{*}\right| \\
& \Longrightarrow \begin{cases}=0 & \text { if } R^{k}>R^{*} \\
<0 & \text { o.w. }\end{cases}
\end{aligned}
$$

Using similar arguments we can analyze the case for $R^{k+1}<$ $R^{*}$ and obtain also that $e\left(\mathbf{r}_{g}^{k+1}\right) \leq e\left(\mathbf{r}_{g}^{k}\right)$. If at step $k+1$ a batch update occurs, (15) and (40) hold for each $i \in \mathscr{M}$. Suppose $R^{k+1} \geq R^{*}$. If there exists an $i \in \mathscr{M}$ such that $r_{i}^{k+1}>r_{i}^{*}$ then part (b) in Assumption 1 implies

$$
\bar{\gamma}_{i}^{k+1}-\underline{\gamma}_{i}^{k+1}<\bar{\gamma}_{i}^{*}-\underline{\gamma}_{i}^{*}
$$

therefore by (26) $R_{g}^{*} \geq R_{g}^{k+1}$. If no such $i$ exists, namely, $r_{i}^{k+1} \leq$ $r^{*}$ for each $i \in \mathscr{M}$, then naturally $R_{g}^{k+1} \leq R_{g}^{*}$. A similar result can be derived for the case $R^{k+1} \leq R^{*}$. We then have

$$
\begin{aligned}
& R^{k+1} \geq R^{*} \Longrightarrow R_{g}^{k+1} \leq R_{g}^{*}, \\
& R^{k+1} \leq R^{*} \Longrightarrow R^{k+1} \geq R_{g}^{*} .
\end{aligned}
$$


TABLE 1: The parameters of $\alpha_{i}$ for $i \in \mathcal{N}=\{1,2, \ldots, 100\}$.

\begin{tabular}{lllllllllll}
\hline$i$ & \multicolumn{1}{c}{0} & \multicolumn{7}{c}{$\alpha_{i}$} & \\
\hline $1-10$ & .6111 & .7052 & .0283 & .5209 & .2591 & .5082 & .9732 & .8182 & .8588 & .8966 \\
$11-20$ & .2995 & .8800 & .2552 & .1921 & .1866 & .8611 & .4446 & .3866 & .8320 & .2065 \\
$21-30$ & .8978 & .4595 & .1634 & .2687 & .5207 & .7724 & .3874 & .0444 & .9868 & .5230 \\
$31-40$ & .8437 & .8919 & .6856 & .5178 & .4420 & .3801 & .0318 & .2159 & .0853 & .7969 \\
$41-50$ & .4018 & .0498 & .3165 & .8712 & .9314 & .4257 & .8662 & .4806 & .9083 & .2505 \\
$51-60$ & .5266 & .2448 & .8384 & .5257 & .3498 & .2571 & .1688 & .5992 & .8110 & .8489 \\
$61-70$ & .8448 & .2350 & .5083 & .6694 & .0907 & .5430 & .2980 & .3818 & .5339 & .4265 \\
$71-80$ & .2320 & .0284 & .0260 & .2743 & .6945 & .3300 & .7505 & .7957 & .0537 & .0092 \\
$81-90$ & .7281 & .6644 & .5853 & .9190 & .1673 & .9720 & .2196 & .0382 & .7078 & .2290 \\
$91-100$ & .3526 & .7095 & .5887 & .1088 & .6468 & .7311 & .8634 & .0226 & .5252 & .1512 \\
\hline
\end{tabular}

Now

$$
\begin{aligned}
e\left(\mathbf{r}_{g}^{k+1}\right)-e\left(\mathbf{r}_{g}^{k}\right)= & \left|R_{g}^{k+1}-R_{g}^{*}\right|-\left|R_{g}^{k}-R_{g}^{*}\right| \\
& +\left|R^{k+1}-R^{*}\right|-\left|R^{k}-R^{*}\right| .
\end{aligned}
$$

Assume $R^{k+1} \geq R^{*}$. If $R^{k} \geq R^{k+1}$ then

$$
\begin{aligned}
e\left(\mathbf{r}_{g}^{k+1}\right)-e\left(\mathbf{r}_{g}^{k}\right) & =-\left(R_{g}^{k+1}-R_{g}^{*}\right)-\left|R_{g}^{k}-R_{g}^{*}\right|+R^{k+1}-R^{k} \\
& \Longrightarrow \begin{cases}=0 & \text { if } R_{g}^{k}<R_{g}^{*} \\
<0 & \text { o.w. }\end{cases}
\end{aligned}
$$

If $R^{k}<R^{k+1}$, which is equivalent to $R_{g}^{k}<R_{g}^{k+1}$, then

$$
\begin{aligned}
e\left(\mathbf{r}_{g}^{k+1}\right)-e\left(\mathbf{r}_{g}^{k}\right) & =-R_{g}^{k+1}+R_{g}^{k}+R^{k+1}-R^{*}-\left|R^{k}-R^{*}\right| \\
& \Longrightarrow \begin{cases}=0 & \text { if } R^{k}>R^{*} \\
<0 & \text { o.w. }\end{cases}
\end{aligned}
$$

Applying similar arguments again we can analyze the case for $R^{k+1}<R^{*}$ and obtain also that $e\left(\mathbf{r}_{g}^{k+1}\right) \leq e\left(\mathbf{r}_{g}^{k}\right)$.

Theorem 10. Algorithm 1 yields a sequence $\left\{\mathbf{r}_{g}^{k}\right\}_{k=1}^{\infty}$ converging to $\mathbf{r}_{g}^{*}$, the user-grouping NEP of the bandwidth allocation game.

Proof. In the light of Lemma 9, we only need to show that for each positive integer $k, \mathbf{r}_{g}^{k} \neq \mathbf{r}_{g}^{*}$ implies the existence of a finite integer $k_{1}$ such that $e\left(\mathbf{r}_{g}^{k+k_{1}}\right)<e\left(\mathbf{r}_{g}^{k}\right)$. Suppose it is not the case then there exists some $k$ such that $e\left(\mathbf{r}_{g}^{k+k_{2}}\right)=e\left(\mathbf{r}_{g}^{k}\right)$ for any positive integer $k_{2}$, where $\mathbf{r}_{g}^{k} \neq \mathbf{r}_{g}^{*}$. Consider the update scheme that, at step $k+i-m$ for $i \in \mathscr{N} \backslash \mathscr{M}$, the individual update occurs at user $i$, and at step $k+n+1-m$ the batch update takes place for users in $\mathscr{M}$. Without loss of generality we assume $R^{k+n+1-m} \geq R^{*}$, then $R_{g}(k+n+1-m) \leq R_{g}^{*}$ by (47). Since $e\left(\mathbf{r}_{g}^{k+n+1-m}\right)=e\left(\mathbf{r}_{g}^{k+n-m}\right)$, (50) together with (51) implies $R^{k+n-m} \geq R^{*}$; hence $R_{g}^{k+n-m} \leq R_{g}^{*}$. That is, $R^{*}-R^{*}$ and $R_{g}-R_{g}^{*}$ do not change their signs as the step number is increased from $k+n-m$ to $k+n+1-m$. Moreover, $R^{k+n-m} \geq$ $R^{*}$ implies $r_{n}^{k+n-m} \leq r_{n}^{*}$. Since $e\left(\mathbf{r}_{g}^{k+n+1-m}\right)=e\left(\mathbf{r}_{g}^{k+i-m-1}\right)$ for $i \in \mathscr{N} \backslash \mathscr{M}$, (44) and (45) together imply $R^{k+i-m} \geq R^{*}$ and thus $r_{i}^{k+i-m} \leq r_{i}^{*}$, for $i \in \mathscr{N} \backslash \mathscr{M}$. As a result, $r_{i}^{k+n-m} \leq r_{i}^{*}$ for $i \in \mathscr{N} \backslash \mathscr{M}$. Note that $R_{g}^{k+n-m} \leq R_{g}^{*}$ and

$$
\begin{aligned}
R^{k+n-m} & =R_{g}^{k+n-m}+\sum_{i=m+1}^{n} r_{i}^{k+n-m} \\
& \geq R^{*}=R_{g}^{*}+\sum_{i=m+1}^{n} r_{i}^{*}
\end{aligned}
$$

We conclude that $R_{g}^{k+n-m}=R_{g}^{*}$ and $r_{i}^{k+n-m}=r_{i}^{*}$ for $i \in \mathscr{N} \backslash \mathscr{M}$, namely, $\mathbf{r}_{g}^{k}=\mathbf{r}_{g}^{*}$, a contradiction.

\section{A Numerical Example}

Consider a data communication network system with 100 users. Suppose 30 of them form a group. We then have $\mathcal{N}=$ $\{1,2, \ldots, 100\}$ and $\mathscr{M}=\{1,2, \ldots, 30\}$. The adopted utility function is

$$
U_{i}\left(r_{i}, R\right)=r_{i}^{\alpha_{i}}(T-R) \text {, }
$$

where the parameters $\alpha_{i}$ 's are listed in Table 1 . Note that the utility function, known as the generalized power function [26], has the continuity and concavity properties required by Assumption 1. In particular, it can be shown [18, page 11] easily that the maximizer

$$
\arg \max _{r} U_{i}(r, R)=\frac{\alpha_{i}}{1+\alpha_{i}} T_{i}<T_{i},
$$

and thus part (a) in Assumption 2 is satisfied. Suppose the total available bandwidth $T=6000$, and the upper and lower bounds for total bandwidth allocated to the group is $\bar{R}_{g}=$ 2000 and $\underline{R}_{g}=800$, respectively. Assume that the individual bandwidth constraint for each user in Table 2 is used. Clearly these parameters satisfy the natural requirements of part (b) in Assumption 2. Applying Algorithm 1 yields a dynamic bandwidth allocation evolving with the implementation step, as shown in Figure 2. The left part of the figure shows the evolution of total bandwidth allocated to the group, which is 
(P1) Initiate the algorithm with a feasible $\mathbf{r}_{g}$ and set $s=0$.

(P2) (if current step is $k$ ) Perform a batch update for users in $\mathscr{M}$ at step $k+1$ by assigning $r_{j}^{k+1}=r_{j}^{k}$ for all $j \in \mathscr{N} \backslash \mathscr{M}$, to reach $r_{i}^{k+1}=\mathscr{A}_{i}\left(T_{i}^{k+1}, T_{i}^{g, k+1}\right)$ for all $i \in \mathscr{M}$.

(P3) (if current step is $\widehat{k}$ ) Perform an individual update at user $i$ in $\mathscr{N} \backslash \mathscr{M}$ by assigning $r_{j}^{\widehat{k}+1}=r_{j}^{\widehat{k}}$ for all $j \in \mathcal{N} \backslash\{i\}$, to reach $r_{i}^{\widehat{k}+1}=A_{i}\left(T_{i}^{\widehat{k}}\right)$, and let $\widehat{k}=\widehat{k}+1$. Sequentially implement this whole procedure till each user in $\mathscr{N} \backslash \mathscr{M}$ is updated.

(P4) Repeat (P3) to complete a batch update at users in $\mathscr{N} \backslash \mathscr{M}$.

(P5) Set $s=s+1$ and record the current $\mathbf{r}_{g}$ as $\mathscr{R}(s)$. If $\|\mathscr{R}(s)-\mathscr{R}(s-1)\|<\varepsilon$ then stops, otherwise go to (P2).

Algorithm 1: The scheme to locate the user-grouping NEP.

Total bandwidth allocation to users in the group

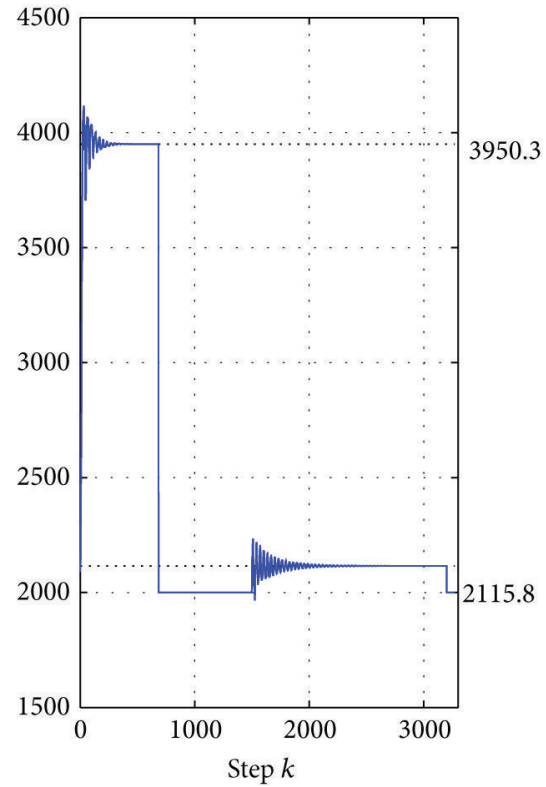

(a)
Bandwidth allocation to users (only 3 users are shown)

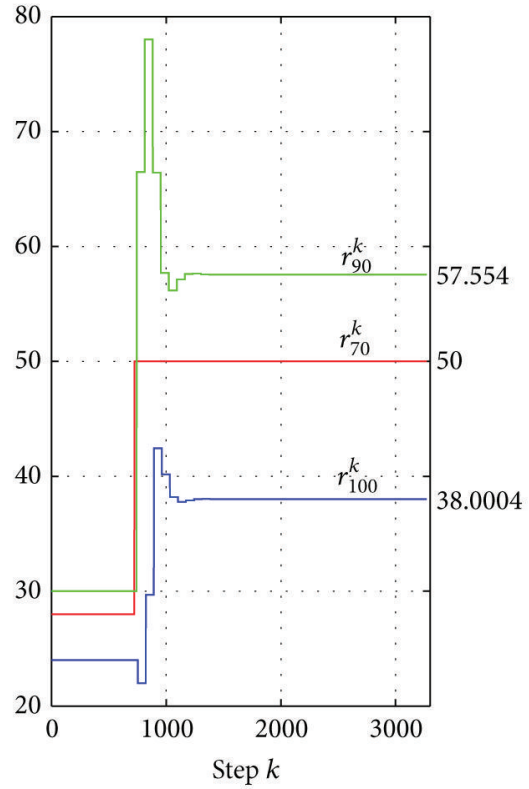

(b)

FIgURE 2: The sequence generated by Algorithm 1 for the example.

composed of user 1, user 2, up to user 30. At the beginning of the algorithm, an initial feasible bandwidth allocation is allocated to each user of the system. Fix the total bandwidth allocated to the users not in the group and find the optimal total bandwidth $R_{g}$. In the example $R_{g}$ is 3950.3. Since this value is greater than the upper bound $\bar{R}_{g}=2000 . R_{g}$ is replaced with $\bar{R}_{g}$. Fix this $R_{g}$ we have the total available bandwidth $T-R_{g}=6000-2000=4000$ for users not in the group. Based on this availability of the bandwidth we can find the equilibrium point for users not in the group. In the example we have, for instance, the bandwidth $r_{70}=$ $50, r_{90}=57.554$, and $r_{100}=38.0004$. Now fix the total bandwidth of the users not in the group, and find the optimal bandwidth $R_{g}$ again. In the example we obtain $R_{g}=2115.8$. Since this value is greater than the upper bound $\bar{R}_{g}=2000$, $R_{g}$ is replaced with $\bar{R}_{g}$ again. Note that the current optimal bandwidth allocation for each user outside the group is found based on the condition that the total bandwidth for the group is $\bar{R}_{g}$. The current $\bar{R}_{g}$ and $r_{m+1}, \ldots, r_{n}$ is thus the $\bar{R}_{g}^{*}$ and $r_{m+1}^{*}, \ldots, r_{n}^{*}$ for the user-grouping NEP in (5).

\section{Conclusion}

We have proposed a novel bandwidth allocation model based on game theory. The consideration of the user-grouping constraint distinguishes this model from the abundant ones 
TABLE 2: The bandwidth constraint for each user.

\begin{tabular}{lcccccc}
\hline User $(i)$ & $1-10$ & $11-20$ & $21-30$ & $31-70$ & $71-90$ & $91-100$ \\
\hline Upper bound $\left(\bar{r}_{i}\right)$ & 100 & 200 & 290 & 50 & 100 \\
Lower bound $\left(\underline{r}_{i}\right)$ & 10 & 20 & 30 & 5 & 10 & 22 \\
\hline
\end{tabular}

concerning similar allocation issues. Suppose each user competes for the system bandwidth resources and is granted with a constrained decision space. In particular, some users are united in one group and the total bandwidth allocated to the group is constrained as well. Given the appropriate constraint parameters and the utility function satisfying mild continuity and concavity conditions for each user, we have shown the unique existence of the user-grouping Nash equilibrium point for the allocation game. In addition, we have shown the fairness, in a proper sense, of the allocation based on this equilibrium point. Finally, we have proposed an iterative algorithm and proved that a sequence converging to the point can be generated by the algorithm. A practical example illustrating a network satisfying our settings has been given to show how the equilibrium point can be located successfully.

\section{Nomenclature}

$\mathcal{N}: \quad$ The index set for the users, that is, $\mathcal{N}:=\{1,2, \ldots, n\}$

$\mathscr{M}$ : The index set for the users in the group, that is, $\mathscr{M}:=\{1,2, \ldots, m\}$

$\mathscr{N} \backslash \mathscr{M}$ : The index set for the users not in the group, that is, $\mathscr{N} \backslash \mathscr{M}:=\{m, m+1, \ldots, n\}$

$r_{i}$ : The bandwidth allocated to user $i$

$\underline{r}_{i}: \quad$ Lower bound for $r_{i}$

$\bar{r}_{i}: \quad$ Upper bound for $r_{i}$

$R_{g}$ : Total bandwidth allocated to the group members, that is, $R_{g}:=r_{1}+r_{2}+\cdots+r_{m}$

$\underline{R}_{g}: \quad$ Lower bound for $R_{g}$

$\bar{R}_{g}$ : $\quad$ Upper bound for $R_{g}$

$T$ : $\quad$ Total bandwidth available in the system

$R: \quad$ Total bandwidth allocated, that is, $R:=r_{1}+r_{2}+\cdots+r_{n}$

$R_{-i}$ : Total bandwidth allocated, excluding to user $i$, that is, $R_{-i}:=r_{1}+\cdots+r_{i-1}+r_{i+1}+$ $\cdots+r_{n}$

$T_{i}$ : Total bandwidth available for user $i$, that is, $T_{i}:=T-R_{-i}$

$T_{i}^{g}: \quad$ Inside-group information for user $i \in \mathscr{M}$, that is, $T_{i}^{g}:=\bar{R}_{g}-\sum_{j \neq i}^{m} r_{j}$.

\section{Acknowledgment}

This research was supported by the National Science Council of Taiwan under Grant NSC 100-2221-E-005-071.

\section{References}

[1] J. Anselmi, U. Ayesta, and A. Wierman, "Competition yields efficiency in load balancing games," Evaluation, vol. 68, no. 11, pp. 986-1001, 2011.
[2] U. Ayesta, O. Brun, and B. J. Prabhu, "Price of anarchy in noncooperative load balancing games," Performance Evaluation, vol. 68, no. 12, pp. 1312-1332, 2011.

[3] R. J. La and V. Anantharam, "Optimal routing control: repeated game approach," IEEE Transactions on Automatic Control, vol. 47, no. 3, pp. 437-450, 2002.

[4] O. Richman and N. Shimkin, "Topological uniqueness of the nash equilibrium for selfish routing with atomic users," Mathematics of Operations Research, vol. 32, no. 1, pp. 215-232, 2007.

[5] T. Boulogne, E. Altman, H. Kameda, and O. Pourtallier, "Mixed equilibrium (ME) for multiclass routing games," IEEE Transactions on Automatic Control, vol. 47, no. 6, pp. 903-916, 2002.

[6] Y. A. Korilis, A. A. Lazar, and A. Orda, "Architecting noncooperative networks," IEEE Journal on Selected Areas in Communications, vol. 13, no. 7, pp. 1241-1251, 1995.

[7] Y. A. Korilis, A. A. Lazar, and A. Orda, "Achieving network optima using Stackelberg routing strategies," IEEE/ACM Transactions on Networking, vol. 5, no. 1, pp. 161-173, 1997.

[8] Y. A. Korilis, A. A. Lazar, and A. Orda, "Capacity allocation under noncooperative routing," IEEE Transactions on Automatic Control, vol. 42, no. 3, pp. 309-325, 1997.

[9] D. Niyato and E. Hossain, "QoS-aware bandwidth allocation and admission control in IEEE 802.16 broadband wireless access networks: a non-cooperative game theoretic approach," Computer Networks, vol. 51, no. 11, pp. 3305-3321, 2007.

[10] A. Ganesh, K. Laevens, and R. Steinberg, "Congestion pricing and noncooperative games in communication networks," Operations Research, vol. 55, no. 3, pp. 430-438, 2007.

[11] H. Yaïche, R. R. Mazumdar, and C. Rosenberg, "A game theoretic framework for bandwidth allocation and pricing in broadband networks," IEEE/ACM Transactions on Networking, vol. 8, no. 5, pp. 667-678, 2000.

[12] E. Altman, T. Boulogne, R. El-Azouzi, T. Jiménez, and L. Wynter, "A survey on networking games in telecommunications," Computers \& Operations Research, vol. 33, no. 2, pp. 286-311, 2006.

[13] F. Bonomi and K. W. Fendick, "Rate -based flow control framework for the available bit rate ATM service," IEEE Network, vol. 9, no. 2, pp. 25-39, 1995.

[14] D. Niyato and E. Hossain, "Queue-aware uplink bandwidth allocation and rate control for polling service in IEEE 802.16 broadband wireless networks," IEEE Transactions on Mobile Computing, vol. 5, no. 6, pp. 668-679, 2006.

[15] A. A. Lazar, A. Orda, and D. E. Pendarakis, "Virtual path bandwidth allocation in multiuser networks," IEEE/ACM Transactions on Networking, vol. 5, no. 6, pp. 861-871, 1997.

[16] J. W. Evans and C. Filsfils, Deploying IP and MPLS QoS for Multiservice Networks: Theory \& Practice, Morgan Kaufmann, Boston, Mass, USA, 1st edition, 2007.

[17] S. H. Rhee and T. Konstantopoulos, "Decentralized optimal flow control with constrained source rates," IEEE Communications Letters, vol. 3, no. 6, pp. 188-190, 1999. 
[18] S. H. Rhee, Optimal flow control and bandwidth allocation in multiservice networks: decentralized approaches [Ph.D. thesis], The University of Texas at Austin, 1999.

[19] C. M. Assi, Y. Ye, S. Dixit, and M. A. Ali, "Dynamic bandwidth allocation for quality-of-service over ethernet PONs," IEEE Journal on Selected Areas in Communications, vol. 21, no. 9, pp. 1467-1477, 2003.

[20] J. Xie, S. Jiang, and Y. Jiang, "A dynamic bandwidth allocation scheme for differentiated services in EPONs," IEEE Communications Magazine, vol. 42, no. 8, pp. 32-39, 2004.

[21] S. I. Choi and J. D. Huh, "Dynamic bandwidth allocation algorithm for multimedia services over ethernet PONs," ETRI Journal, vol. 24, no. 6, pp. 465-468, 2002.

[22] J. B. Rosen, "Existence and uniqueness of equilibrium points for concave n-person games," Econometrica, vol. 33, pp. 520-534, 1965.

[23] J. F. Nash, "Equilibrium points in $n$-person games," Proceedings of the National Academy of Sciences of the United States of America, vol. 36, pp. 48-49, 1950.

[24] J. Nash, "Non-cooperative games," Annals of Mathematics, Second Series, vol. 54, pp. 286-295, 1951.

[25] E. K. P. Chong and S. H. Zak, An Introduction to Optimization, Wiley-Interscience, Hoboken, NJ, USA, 3rd edition, 2008.

[26] A. Bovopoulos and A. Lazar, "Decentralized algorithms for optimal flow control," in Proceedings of the 25th Allerton Conference on Communication, Control, and Computing, pp. 979-988, 1987. 


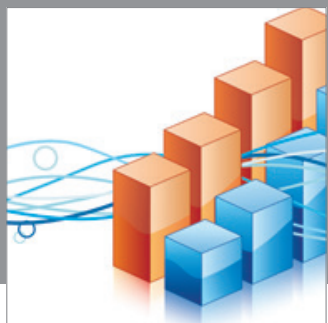

Advances in

Operations Research

mansans

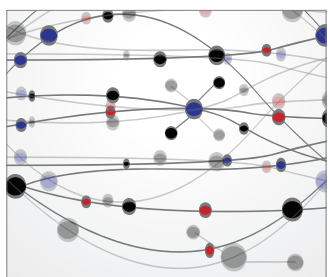

The Scientific World Journal
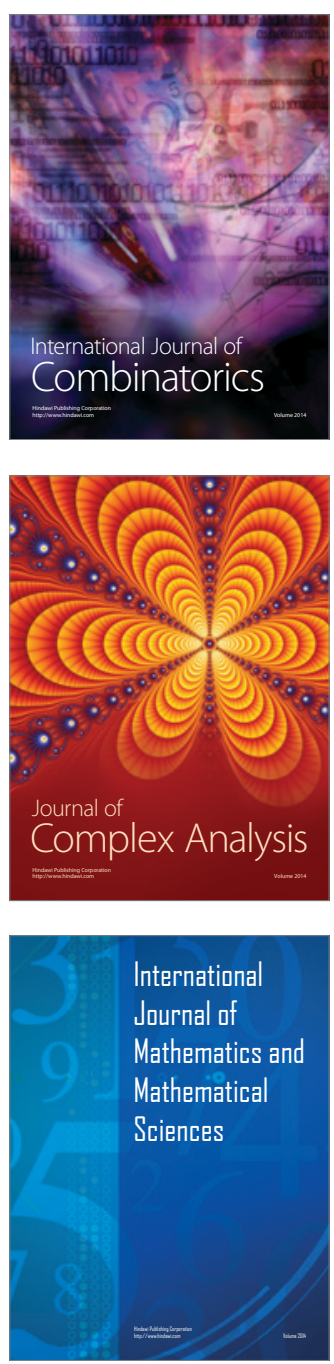
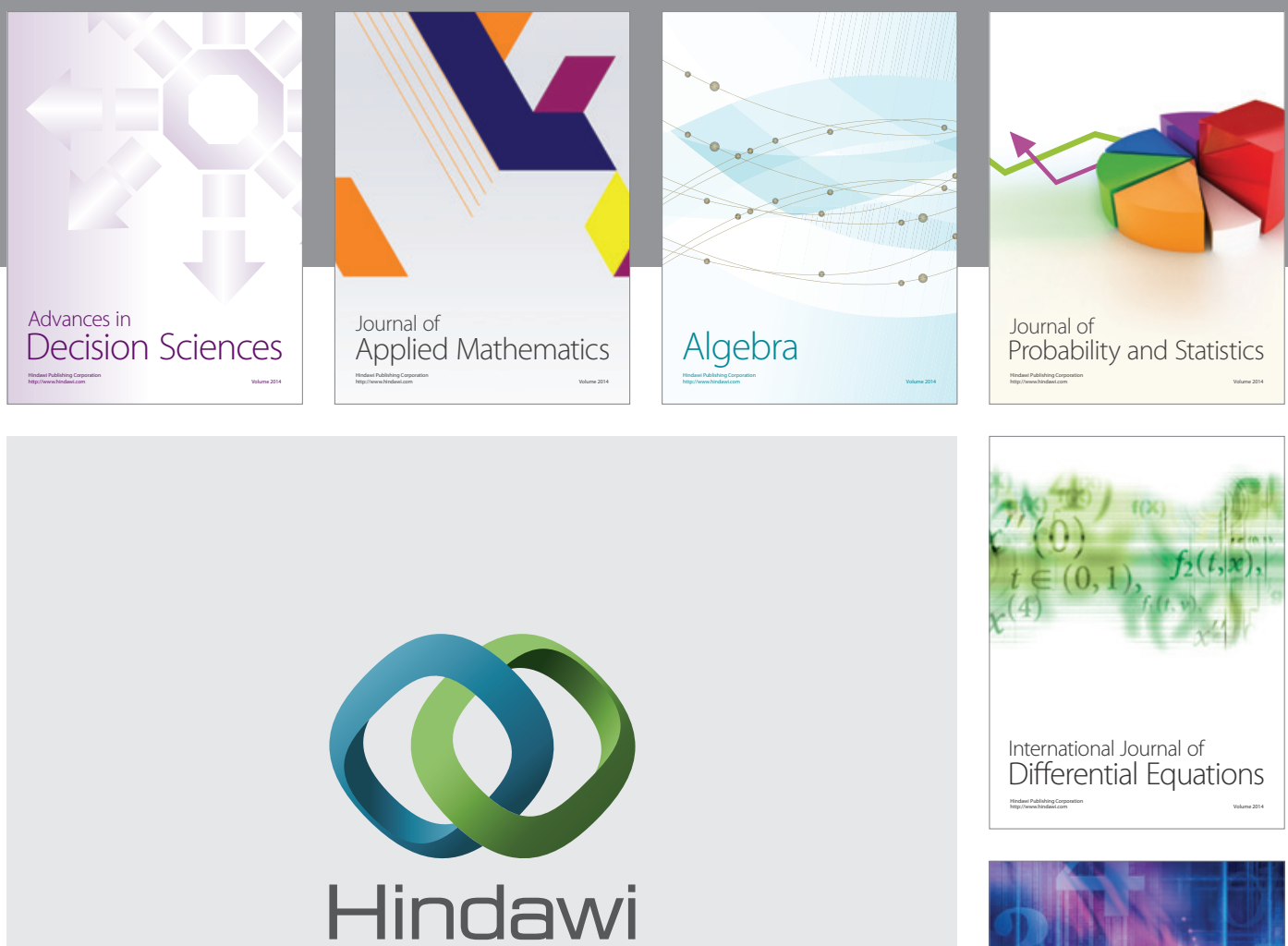

Submit your manuscripts at http://www.hindawi.com
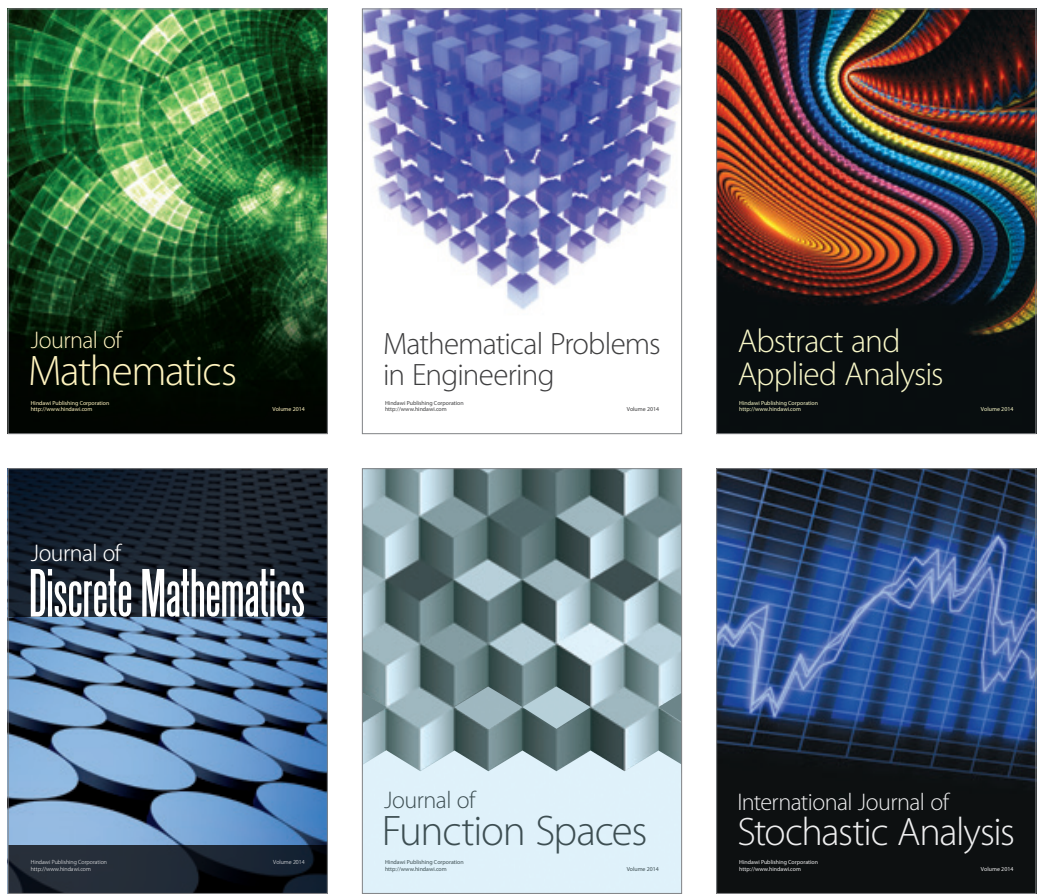

Journal of

Function Spaces

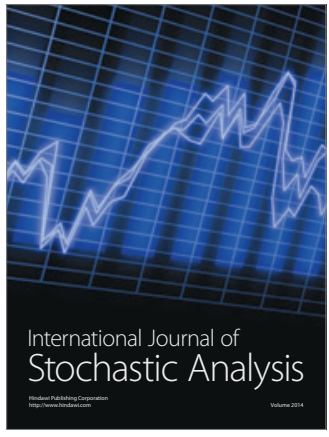

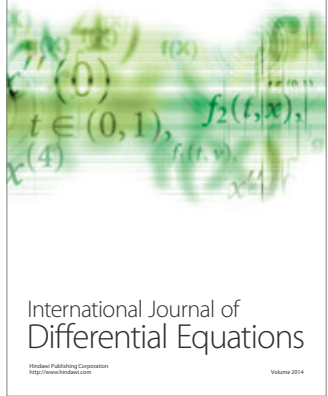
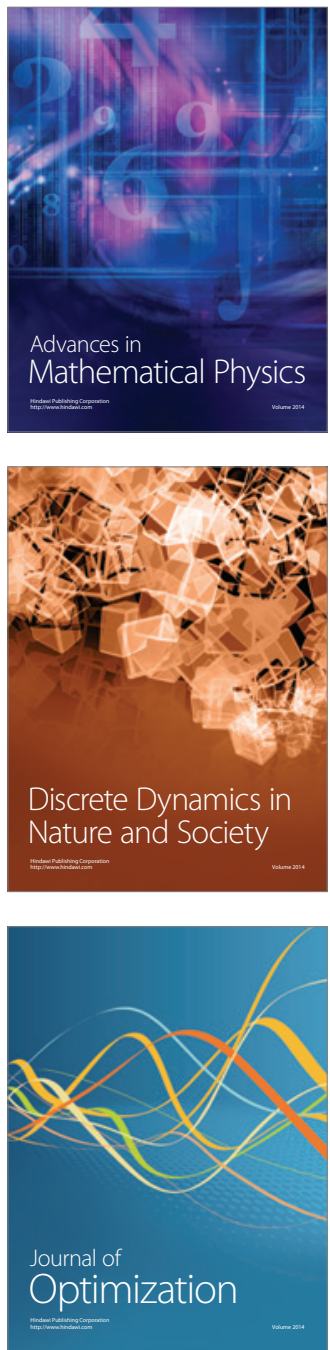